\title{
Fermi/LAT observations of Dwarf Galaxies highly constrain a Dark Matter Interpretation of Excess Positrons seen in AMS-02, HEAT, and PAMELA
}

\author{
Alejandro Lópezt \\ Michigan Center for Theoretical Physics, University of Michigan, Ann Arbor, Michigan 48109-1040, USA \\ Christopher Savag甲 \\ Nordita (Nordic Institute for Theoretical Physics), \\ KTH Royal Institute of Technology and Stockholm University, \\ Roslagstullsbacken 23, SE-106 91 Stockholm, Sweden \\ Douglas Spolyar \\ Oskar Klein Centre for Cosmoparticle Physics, Stockholm University, Stockholm, Sweden \\ Douglas Q. Adams $\$$ \\ 99 Battery Place, New York, NY 10280, USA
}

(Dated: May 27, 2022)

\begin{abstract}
It is shown that a Weakly Interacting Massive dark matter Particle (WIMP) interpretation for the positron excess observed in a variety of experiments, HEAT, PAMELA, and AMS-02, is highly constrained by the Fermi/LAT observations of dwarf galaxies. In particular, this paper has focused on the annihilation channels that best fit the current AMS-02 data (Boudaud et al., 2014). The Fermi satellite has surveyed the $\gamma$-ray sky, and its observations of dwarf satellites are used to place strong bounds on the annihilation of WIMPs into a variety of channels. For the single channel case, we find that dark matter annihilation into $\left\{b \bar{b}, e^{+} e^{-}, \mu^{+} \mu^{-}, \tau^{+} \tau^{-}, 4-e\right.$, or $\left.4-\tau\right\}$ is ruled out as an explanation of the AMS positron excess (here $b$ quarks are a proxy for all quarks, gauge and Higgs bosons). In addition, we find that the Fermi/LAT $2 \sigma$ upper limits, assuming the best-fit AMS-02 branching ratios, exclude multichannel combinations into $b \bar{b}$ and leptons. The tension between the results might relax if the branching ratios are allowed to deviate from their best-fit values, though a substantial change would be required. Of all the channels we considered, the only viable channel that survives the Fermi/LAT constraint and produces a good fit to the AMS-02 data is annihilation (via a mediator) to $4-\mu$, or mainly to $4-\mu$ in the case of multichannel combinations.
\end{abstract}

Weakly Interacting Massive Particles (WIMPS), such as the lightest supersymmetric particles (for reviews, see Refs. 1, 2]), are thought to be the best motivated dark matter (DM) candidates. The particles in consideration are their own antiparticles; thus, they annihilate among themselves in the early universe and naturally provide the correct relic density today to explain the dark matter of the universe. This same annihilation process takes place in the present universe wherever the DM density is sufficiently high and is the basis for DM indirect detection searches. Indirect detection experiments search for the annihilation products of dark matter particles, including electrons and/or positrons, antiprotons, photons, and neutrinos. Promising sites for the observation of dark matter annihilation products include the core of the Sun [3], the Earth [4, 5], our Galactic halo [6] 9], Galactic center [10, dwarf satellite galaxies [1, 12, and from DM substructures [13 16.

Over the past several years, there have been a number of experimental signals which have been interpreted as possible indications of DM. Confirmation that any of these observations are actually due to DM, rather than being a mere experimental artifact or astrophysical background, would likely require more than one experiment to provide complementary information. In this paper, we consider the anomalous features in the spectrum of cosmic ray positrons and electrons reported by AMS-02 [17, PAMELA [18, 19, and the Large Area Telescope of the Fermi Gamma Ray Space Telescope (Fermi/LAT) [20] (as well as in earlier indications from HEAT [21 23]). The positron fraction was found to be a steadily increasing function of energy, above $10 \mathrm{GeV}$. This behavior is difficult to explain with standard astrophysical mechanisms, in which positrons are secondary particles, produced in the interactions of primary cosmic rays during the propagation in the interstellar medium. These observations have led to a great deal of speculation that DM annihilations [24-43] or decays [44 47. may be responsible. However, any explanation of these positron/electron signals in terms of DM annihilation requires somewhat nonstandard WIMP properties. In particular, the local

\footnotetext{
*Electronic address: aolopez@umich.edu

† Electronic address: chris@savage.name

$\ddagger$ Electronic address: dspolyar@gmail.com

$\S$ Electronic address: doug.q.adams@gmail.com
} 
halo density of DM within the vicinity of the Solar System is insufficient to produce these observations unless the annihilation cross section is considerably larger than that typically expected for a thermal relic, or the annihilation rate is otherwise supplemented by a large boost factor $\sim 10^{1}-10^{4}$. Such an enhancement could arise due to astrophysics; for example, due to substructures in the DM distribution; yet Ref. [48 argues that the probability of such a nearby DM clump is $<1 \%$. In this paper, we will instead focus on the possibility of an annihilation cross-section that is enhanced by the required boost factor compared to the standard thermal annihilation. Furthermore, only a handful of possibilities can explain the spectral shape reported by AMS-02 and PAMELA, as well as avoid overproducing antiprotons (in excess of what is observed) 49 52. The most well-studied approach to satisfying all these constraints has been leptophilic DM, i.e. the DM annihilations proceed largely to leptons $\left(\mu^{+} \mu^{-}\right.$or $\left.\tau^{-} \tau^{-}\right)$, which do not produce any antiprotons. Alternatively, other annihilations channels - quarks, vector and Higgs bosons - are allowed if the DM particle is heavier than $\sim 10 \mathrm{TeV}$, producing antiprotons at higher energies than those probed by AMS-02 data [27.

Other explanations of the Cosmic Ray Positron Excess (henceforth CRPE) have also been proposed. The most plausible is that it is due to pulsars [53 56].

Positrons can ultimately be produced by DM annihilating into a variety of channels: single channels (leptons, quarks, gauge bosons, Higgs, or four leptons) or a mix of these channels. Prior to 2014, when the most recent AMS-02 results became available, all annihilation channels could provide explanations for the positron excess (see Table 2 in Ref. [57]). The most popularly studied cases were WIMP masses $\sim 200 \mathrm{GeV}$, which matched the data only for leptophilic channels (DM annihilates only into leptons $\mu^{+} \mu^{-}$or $\tau^{+} \tau^{-}$), though quark and gauge boson channels well fit the data at higher WIMP masses, with best-fit masses as high as $\sim 50-200 \mathrm{TeV}$ (depending on the channel).

The recent AMS-02 data release [17] has greatly improved our understanding of the positron excess. As stressed by Ref. [57, two major improvements have emerged. First the new data are far more accurate and extend out to $500 \mathrm{GeV}$, much higher energies than previously explored. Improved accuracy in the positron spectrum leads to stronger constraints on any model for the origin of the positrons. Second, AMS-02 has measured directly the total electron and positron flux, the denominator in the positron fraction, reducing systematic errors and leading to differences in best-fit regions for the DM mass and cross section. Ref. [57 further stressed the importance of addressing uncertainties in the cosmic ray $(\mathrm{CR})$ propagation model, as these models are of critical importance in assessing the true nature of the positron excess.

Boudaud et al. 57] have performed an analysis with the new AMS-02 data, exploring the DM models that could explain the CRPE. Compared to earlier data and analyses, the recent AMS-02 data do indeed lead to much stronger constraints on suitable DM candidates that can explain the $e^{+}$excess and the best-fit masses have shifted by a fair amount. Primarily these authors used the benchmark set of CR propagation model parameters known as MED in obtaining their results; this is the model that best fits the $\mathrm{B} / \mathrm{C}$ ratio in the cosmic rays. With the recent, more accurate data and using MED, these authors found that many of the earlier allowed models have been ruled out (see Table 1 in Ref. [57]). In terms of single channel models, leptophillic DM is no longer viable, whereas single channel annihilation into quarks and gauge bosons provide an excellent fit to the CRPE. They also considered the case of DM annihilation into a mediator particle $\phi$, where $\chi \chi \rightarrow \phi \phi$ with $\phi \rightarrow \ell \bar{\ell}$, thus yielding four leptons per annihilation ("4-lepton" channel). Again, single channel annihilation into four leptons does not fit the AMS-02 data (assuming MED propagation). Alternatively, annihilation into a combination of channels can also provide an excellent fit, in particular annihilation into an admixture of leptons and $b \bar{b}$ pairs. Finally, a combination of four lepton channels (arising from a mediator field $\phi$ ), specifically the four-tau (75\%) and four-electron (25\%) channel, turns out to provide a good fit to the AMS-02 data for a DM mass between 0.5 and $1 \mathrm{TeV}$. The results reprised in this paragraph all refer to the MED CR propagation model.

Far larger than the statistical errors in the AMS-02 data are the systematic errors associated with the CR propagation. Thus Boudaud et al. [57] looked at a set of 1623 different combinations of the CR transportation parameters-all consistent with observed boron-to-carbon ratios - which bracket the systematic uncertainty in the propagation model (see also Ref. [58 for an analysis of the new AMS-02 data, with a different treatment of the propagation). By including these propagation model parameters into the fits to the AMS-02 data, specifically by finding the DM parameters that fit the data for any of the 1623 propagation parameter combinations, the allowed DM parameter space increases. In terms of the single channel models, the $2-\tau, 4-\mu$, and $4-\tau$ cases can now provide excellent fits to the positron excess in addition to the cases mentioned above.

DM annihilations that produce $e^{+}$, either directly or through decays and showering of the primary annihilation products, will invariably also produce $\gamma$-rays. Thus we can constrain the above DM models by comparing with $\gamma$-ray observations. The best places to look are regions with a large abundance of DM: the Galactic Center, clusters, and dwarf galaxies. We will focus on dwarf galaxies for the remainder of the paper. The dwarf spheroidal galaxies inside the Milky Way are some of the most dark matter dominated objects known, with mass-to-light ratios as high as 1000. Because they are so dark matter rich and nearby, they are exceptionally good places to indirectly detect DM via $\gamma$-rays produced in its annihilation. For previous $\gamma$-ray data that could be used to constrain a DM interpretation of a 
positron excess, see the previous Fermi/LAT combined analysis of dwarf galaxies [59], VERITAS [60], and MAGIC [61] observations of Segue 1, as well as results of the H.E.S.S. collaboration [62 on Sagittarius and other dwarf galaxies.

Fermi/LAT surveys the $\gamma$-ray sky and, specifically, has looked for $\gamma$-rays from 25 dwarf galaxies, detecting no significant excess [59]. This lack of signal is used to place $\gamma$-ray flux upper limits for energies between $500 \mathrm{MeV}$ and $500 \mathrm{GeV}$. These bounds are then used to constrain the dark matter annihilation for a broad range of particle masses and annihilation channels.

The differential $\gamma$-ray flux $\frac{d \phi}{d E}$ from DM annihilation in a dwarf galaxy can be written as the product of two components, a factor $\frac{d \Phi}{d E}$ that encodes all the particle physics and the so-called J-factor that contains the astrophysics,

$$
\frac{d \phi}{d E}=\frac{d \Phi_{\mathrm{pp}}}{d E} \times J=\left(\frac{1}{8 \pi} \frac{\langle\sigma v\rangle}{m_{\chi}^{2}} \frac{d N}{d E}\right) \times\left(\int_{\Delta \Omega} \int_{\text {l.o.s. }} \rho^{2}(r) d r d \Omega\right)
$$

Here, $\langle\sigma v\rangle$ is the DM annihilation cross section, $m_{\chi}$ is the DM mass, and $\frac{d N}{d E}$ is the photon spectrum from the DM annihilation, which depends on the DM mass $m_{\chi}$ and the annihilation channel. The J-factor (the term in the second set of parentheses) integrates the square of the DM density $\rho_{\chi}$ along the line of sight and over a solid angle $\Delta \Omega$. The J-factor can be estimated from stellar kinematics as stars act as tracers of the gravitational potential, allowing the DM distribution to be inferred.

We use Fermi/LAT dwarf $\gamma$-ray results to constrain DM models using the data and likelihood technique described by the Fermi/LAT collaboration in Ref. [59], to be briefly reviewed here. The primary quantity used in the likelihood analysis is the energy flux

$$
s_{k, j}=\int_{E_{j, \min }}^{E_{j, \max }} E \frac{d \phi_{\mathrm{k}}}{d E} d E
$$

for each dwarf (indexed by $k$ ) and energy bin (indexed by $j$ ). Here, $\frac{d \phi_{\mathrm{k}}}{d E} \equiv \frac{d \Phi_{\mathrm{pp}}}{d E} \times J_{k}$ is the differential flux for a dwarf with J-factor $J_{k}$. For each dwarf and energy bin, Fermi/LAT provides a likelihood $\mathcal{L}_{k, j}$ in $s_{k, j}$. The likelihood function accounts for instrument performance, the observed counts, exposure, and background fluxes. For a given annihilation channel, the energy flux is dependent only on the theoretical parameters $m_{\chi},\langle\sigma v\rangle$, and $J_{k} ;$ i.e. $s_{k, j}=s_{k, j}\left(m_{\chi},\langle\sigma v\rangle, J_{k}\right)$.

Accounting for observational constraints on the J-factor, the likelihood for a given dwarf $\mathcal{L}_{k}$ is

$$
\mathcal{L}_{k}\left(m_{\chi},\langle\sigma v\rangle, J_{k}\right)=\mathcal{L} \mathcal{N}\left(J_{k} \mid \bar{J}_{k}, \sigma_{k}\right) \prod_{j} \mathcal{L}_{k, j}\left(s_{k, j}\left(m_{\chi},\langle\sigma v\rangle, J_{k}\right)\right)
$$

where $\mathcal{L} \mathcal{N}$ represents a log-normal distribution and $\bar{J}_{k} \& \sigma_{k}$ are the parameters describing that distribution, derived from the stellar kinematics in the dwarf. The combined likelihood for multiple dwarfs is

$$
\mathcal{L}\left(m_{\chi},\langle\sigma v\rangle, \mathbf{J}\right)=\prod_{k} \mathcal{L}_{k}\left(m_{\chi},\langle\sigma v\rangle, J_{k}\right)
$$

where $\mathbf{J}$ represents the set of J-factors $\left\{J_{k}\right\}$, one for each dwarf. We use in our analysis the same 15 non-overlapping dwarf galaxies with J-factor estimates that are used by Fermi/LAT in their analysis. We use the J-factor estimates based upon a Navarro-Frank-White (NFW) density profile [63], though a Burkert profile [64] would not significantly affect our results. ${ }^{1}$

Fermi/LAT constraints in $\langle\sigma v\rangle$ at a given $m_{\chi}$ are determined using a delta-log-likelihood approach treating the J-factors as nuisance parameters. The delta-log-likelihood $\Delta \mathcal{L}$ is given by

$$
\Delta \ln \mathcal{L}\left(m_{\chi},\langle\sigma v\rangle\right) \equiv \ln \mathcal{L}\left(m_{\chi},\langle\sigma v\rangle, \widehat{\widehat{J}}\right)-\ln \mathcal{L}\left(m_{\chi}, \widehat{\langle\sigma v\rangle}, \widehat{\mathbf{J}}\right)
$$

where $\widehat{\langle\sigma v\rangle} \& \widehat{\mathbf{J}}$ are the values of $\langle\sigma v\rangle \& \mathbf{J}$ that jointly maximize the likelihood at the given $m_{\chi}$ and $\widehat{\widehat{\mathbf{J}}} \equiv \widehat{\widehat{\mathbf{J}}}\left(m_{\chi},\langle\sigma v\rangle\right)$ are the J-factors that maximize the likelihood for the given $m_{\chi}$ and $\langle\sigma v\rangle$. The $1 \mathrm{D}$ confidence intervals in $\langle\sigma v\rangle$ at the $n \sigma$ confidence level are determined by identifying the range of $\langle\sigma v\rangle$ such that

$$
\Delta \ln \mathcal{L}\left(m_{\chi},\langle\sigma v\rangle\right) \leq n^{2} / 2
$$

1 References [59, 65] have shown that the the integrated J-factor within 0.5 degrees is fairly insensitive to the choice of dark matter density profile so long as the central value of the slope is less than 1.2. 

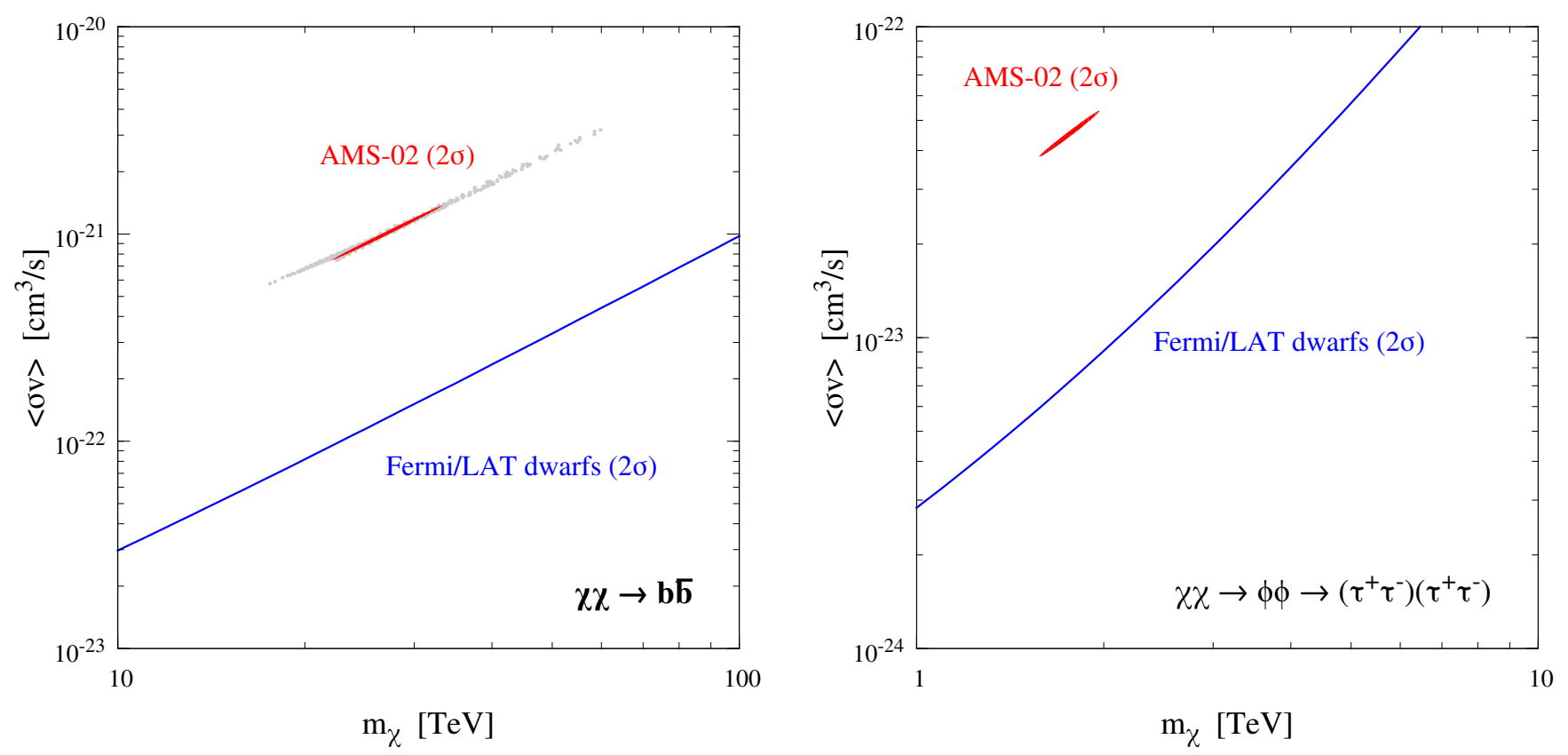

FIG. 1: Constraints on the dark matter annihilation cross-section $\langle\sigma v\rangle$ and mass $m_{\chi}$ for annihilations into $b \bar{b}$ (left) and four $\tau$ 's via a mediator $\phi$ (right). The best-fit AMS-02 parameters, as derived by Ref. [57] for the MED propagation model parameters, are shown in red, while the Fermi/LAT upper bounds are shown by the blue curves. Fermi/LAT dwarf constraints are generated using the procedure described in Ref. [59]. Constraints are shown at the $2 \sigma$ confidence level. For the $b \bar{b}$ case, the AMS-02 best-fit points for a selection of other cosmic ray (CR) propagation model parameters are shown as gray dots (also taken from Ref. [57]).

We will generally show the upper limit of the $2 \sigma$ confidence intervals (95.4\% confidence level).

We consider the case of DM annihilating directly to $b \bar{b}$ and leptons $\ell \bar{\ell}$, as well as the "4-lepton" channels (via a mediator $\phi$ ). We use the $b \bar{b}$ case as a proxy for all other quarks, gauge bosons, and the Higgs boson. The spectra for the $u, d, c, s$, and $t$ quarks; the $W, Z$, and $g$ gauge bosons; and the $H$ boson are all similar in shape to the $b$ spectrum and within $\sim 50 \%$ of the amplitude. Such differences of at most a factor of two in amplitude will not prove to be significant; thus the $b$ spectrum should be reasonably representative of these other cases. ${ }^{2}$ The spectra from leptons, on the other hand, depend on the flavor, so we consider $e, \mu$, and $\tau$ separately. The annihilation spectra $\frac{d N}{d E}$ for these different channels are derived using PYTHIA8 [66, 67. For the first set of cases, annihilation directly to quarks or leptons, we include final state radiation (FSR) in the PYTHIA simulations as these are the primary source of photons for the $e^{+} e^{-}$and $\mu^{+} \mu^{-}$channels; the FSR provides only a minor contribution to the $b \bar{b}$ and $\tau^{+} \tau^{-}$channels. We do not include FSR for the mediator case due to the complexity, though that is again the primary source of photons for the $4-e$ and $4-\mu$ channels.

The AMS-02 $2 \sigma$ confidence regions in $\langle\sigma v\rangle$ vs. $m_{\chi}$ for the $b \bar{b}$ and 4- $\tau$ channels are shown in red in the left and right panels, respectively, of Figure 1, taken from Ref. [57. The Fermi/LAT $2 \sigma$ upper limits in $\langle\sigma v\rangle$ are shown by the blue curves in the figure. For both channels, the AMS-02 regions are strongly excluded by the Fermi/LAT dwarfs data. In Figure 1, the AMS-02 $2 \sigma$ confidence regions were determined assuming the MED propagation parameters, the set of five astrophysical parameters fixed to best fit the measured B/C ratio [68, 69. As the choice of propagation parameters has an impact on the fit to the AMS-02 data, we show also the best-fit points found by Ref. [57 for a selection of other CR propagation model parameters as discussed above. These best-fit points, shown as gray dots for the $b \bar{b}$ case, are taken from Figure 14 of Ref. [57] (parameters with $p \geq 0.0455$ only). Though varying the propagation parameters broadens the region of parameter space consistent with the AMS-02 data, the DM interpretation of the positron spectrum remains strongly in conflict with the Fermi/LAT dwarf $\gamma$-ray observations.

We wish to comment on the differences between regions compatible with AMS-02 assuming the MED propagation

2 Each of the AMS-02 and Fermi constraints vary by less than a factor of two from the $b \bar{b}$ case when looking at other quarks, but $b \bar{b}$ is ruled out by an order of magnitude, so a factor of two is not enough to evade the Fermi/LAT constraints. 


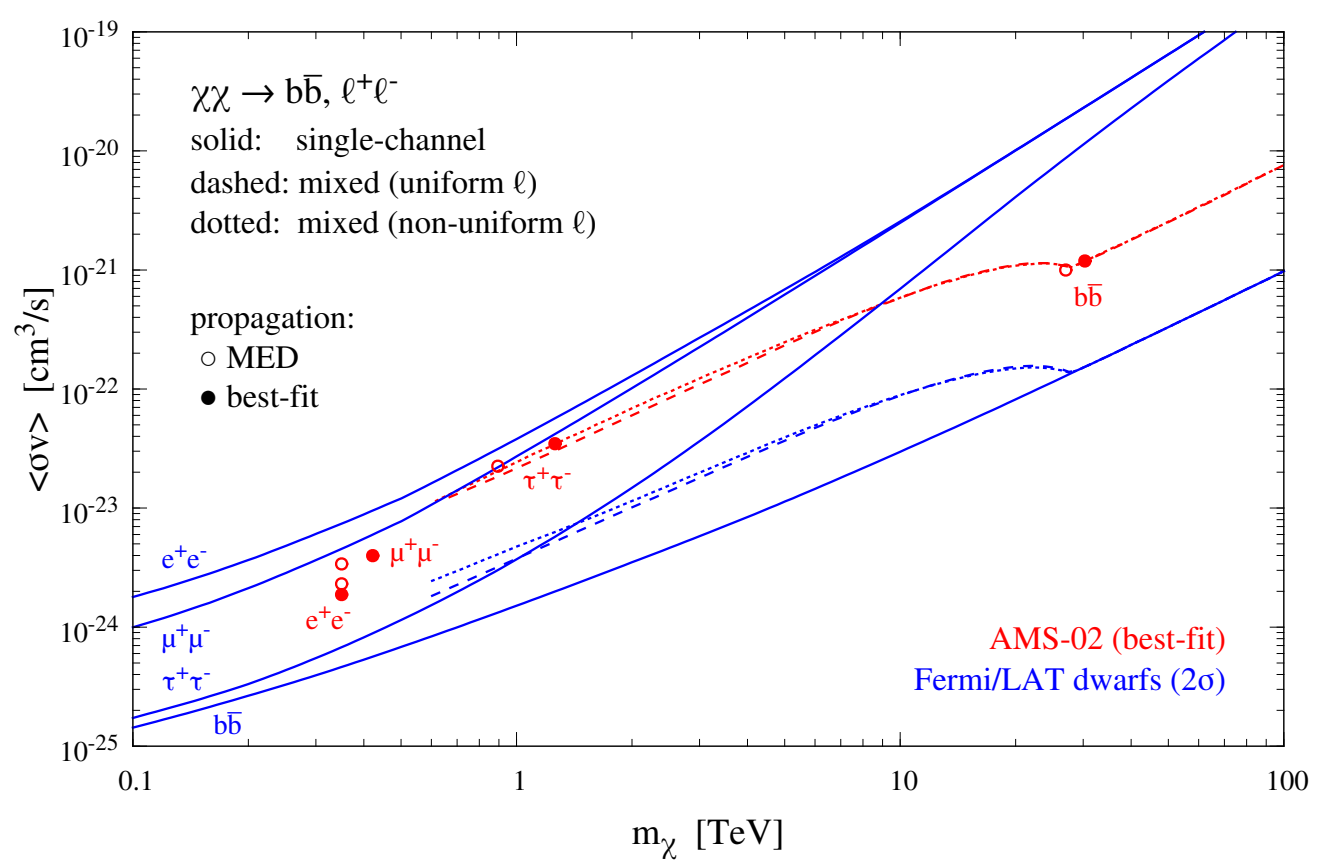

FIG. 2: AMS-02 best-fit parameters and Fermi/LAT constraints on $\langle\sigma v\rangle$ and $m_{\chi}$ for the $b \bar{b}$ and $\ell \bar{\ell}$ channels. Best-fit AMS02 values for single annihilation channels are shown as red circles (taken from Ref. [57]). Empty circles are for the MED propagation parameters and filled circles are for the best-fit propagation parameters. Fermi/LAT constraints for each of these single channels are shown as solid blue lines. The red and blue dashed curves represent respectively the AMS-02 best-fit and Fermi/LAT constraint on $\langle\sigma v\rangle$ at each mass for the mixed $b \bar{b}+\ell \bar{\ell}$ channels, assuming a common (uniform) branching ratio (BR) into each of the three leptons; Fermi/LAT constraints are generated assuming the AMS-02 best-fit BRs at each mass. The dotted curves are the same, but relaxing the assumption of uniform lepton BRs. We note that the MED propagation parameters are used for the mixed channels. While we do not believe that changing the CR propagation parameters will significantly affect our results, proving this assertion is beyond the scope of this paper.

parameters and the regions compatible when the propagation parameters are allowed to vary over reasonable values, as represented by the 1623 sets of CR propagation parameters. In Figure 1 one can see that all the points, for both MED and other CR propagation model parameters, are in an elongated region roughly parallel to the Fermi/LAT bounds. Thus changing the CR propagation parameters does not alleviate the tension between Fermi/LAT and a DM interpretation of AMS-02. We believe that this statement will be generally true, not just for the $b \bar{b}$ channel but for all channels (single and mixed). ${ }^{3}$ Thus we believe the following: if a DM annihilation channel that fits AMS-02 with MED propagation is ruled out by Fermi/LAT, then we believe that the same channel is also likely to be ruled out if other propagation parameters are used. In other words, we do not believe that using different reasonable CR propagation parameters will change our results. However, we cannot prove this assertion without a detailed reanalysis of the AMS-02 data, beyond the scope of the current paper.

Figure 2 shows the AMS-02 best fits and Fermi/LAT dwarf constraints on $\langle\sigma v\rangle$ and $m_{\chi}$ for annihilations into single channel $\bar{\ell}$ or $b \bar{b}$, as well as mixed cases $\ell \bar{\ell}+b \bar{b}$. Note that Figure 2 is over a broader mass range than Figure 1 . First let us discuss the single channel cases. The Fermi/LAT upper bounds on the single channel cases are shown as solid blue lines, from bottom to top: $b \bar{b}, \tau^{+} \tau^{-}, \mu^{+} \mu^{-}, e^{+} e^{-}$. The $e$ and $\mu$ channels have weaker limits as photon production is suppressed in these cases, here coming only from the FSR. ${ }^{4}$ The $b$ and $\tau$ channels produce photons through unsuppressed shower/decay processes; the presence of FSR has little impact on the constraints for these two channels. The best-fit AMS-02 points for these annihilation channels are shown by the red circles. Empty circles are for the MED propagation parameters and filled circles are for the best-fit propagation parameters [57]; the DM

\footnotetext{
${ }^{3}$ Since the annihilation rate scales as $\langle\sigma v\rangle / m_{\chi}^{2}$, moving along the elongation line corresponds to roughly a fixed number of $e^{+}$, as is required to match the AMS-02 data. There is a slight effect on the fit due to the $m_{\chi}$ dependence of the spectrum, but the general trend still holds.

${ }^{4}$ The muon produces a photon in $O(1 \%)$ of its decays, though this process is not accounted for in PYTHIA.
} 


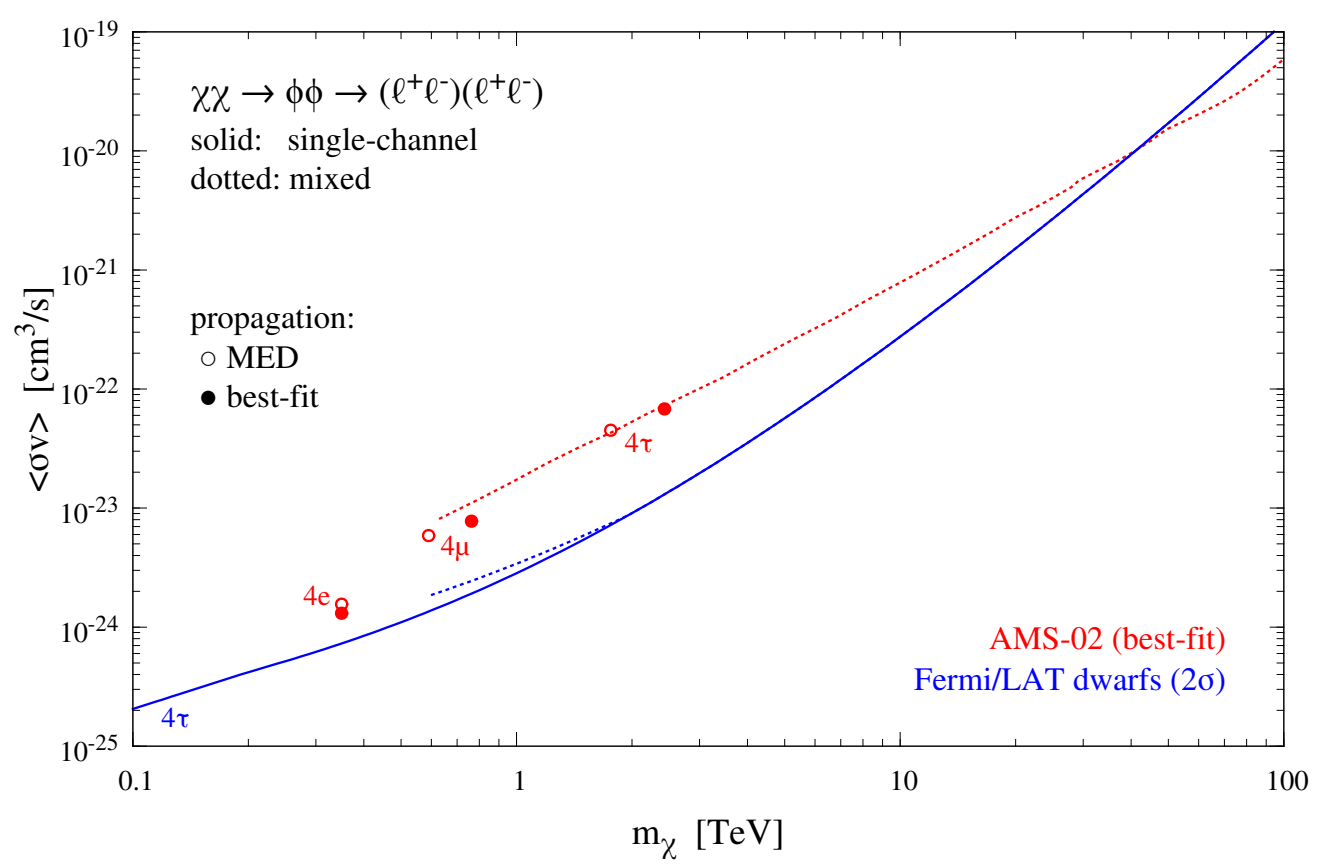

FIG. 3: Same as Figure 2, but for the mediated 4-lepton channels. The solid blue line is the Fermi/LAT bound for the 4- $\tau$ case, which excludes the best-fit AMS-02 values. The 4-e and 4- $\mu$ Fermi/LAT bounds have not been calculated here, but are likely to be weaker than their corresponding best-fit AMS-02 parameters (see the text). Whereas the 4-e case does not provide a good fit to the AMS-02 data, the 4- $\mu$ case does and thus survives as a viable alternative.

parameters do not differ much between the two cases for any of the channels. The AMS- $02 b$ and $\tau$ best-fit points are excluded by the Fermi/LAT constraints, while the $e$ and $\mu$ points are not. The two lighter lepton channels fail to be excluded by Fermi/LAT because they provide positrons in abundance for the AMS-02 signal, while photon production is suppressed, leading to little expected sensitivity to these channels via $\gamma$-ray searches. However, both the $e$ and $\mu$ annihilation channels, while capable of producing substantial numbers of positrons, are simply a poor fit to the AMS-02 spectrum. Thus, none of these four channels can provide a reasonable fit to both the AMS-02 and Fermi/LAT results and no single annihilation channel into quarks, leptons, or gauge bosons can simultaneously explain the AMS-02 data while remaining in agreement with Fermi-LAT bounds. We note that both the MED and the best-fit CR propagation parameters have been used in studying these single channel cases.

Reference [57] also considered the mixed-channel case, where the DM annihilates into some combination of $b \bar{b}$ and the three leptons. Here the MED propagation model is used for the mixed channels. Two possibilities were considered: one where all three leptons were assumed to have a common (uniform) branching ratio (BR) and one where this assumption is relaxed. The AMS-02 best-fit $\langle\sigma v\rangle$ as a function of mass are shown as red dashed and dotted curves for the uniform and non-uniform lepton cases, respectively (taken from Figures $7 \& 5$ in their paper). Their results were presented only down to DM masses of $0.6 \mathrm{TeV}$, hence the termination of the curves at that mass. For the uniform case, the leading annihilation channel is always to $b \bar{b}$, while for the non-uniform case, $\tau^{+} \tau^{-}$dominates below $20 \mathrm{GeV}$ and $b \bar{b}$ dominates above. The corresponding Fermi/LAT $2 \sigma$ upper limits, assuming the best-fit BRs, are shown in blue dashed and dotted curves. The AMS-02 best-fit $\langle\sigma v\rangle$ and BRs are strongly excluded by the Fermi/LAT results. This does not rigorously imply the AMS-02 and Fermi/LAT results are in strong conflict for all multi-channel cases, as the Fermi/LAT constraints will vary if the BRs are allowed to deviate from their best-fit values. However, bringing the two results into compatibility will require the $b \bar{b}$ channel to be heavily suppressed, as well as the $\tau^{+} \tau^{-}$ channel at the lighter end of the mass range, which is quite different from the best-fit case, where $b \bar{b}$ dominates at higher masses and $b \bar{b}+\tau^{+} \tau^{-}$accounts for $\geq 50 \%$ of the annihilations at lower masses.

Figure 3 examines the mediated 4-lepton case with $\chi \chi \rightarrow \phi \phi$ and $\phi \rightarrow \ell \bar{\ell}$. The 4- $\tau$ Fermi/LAT upper limit is shown by the solid blue curve. Due to the complexity of implementing their non-leading-order photon production mechanisms, we have foregone the calculation of limits for the 4-e and 4- $\mu$ cases. However, as with the 2-lepton cases shown in Figure 2, these $e$ and $\mu$ limits can be expected to be 1-2 orders of magnitude weaker than the $\tau$ limit. The AMS-02 best-fit points are again shown by red circles, determined using both MED propagation (empty circles) and the best-fit propagation parameters (solid circles) [57. The $\tau$ point is excluded by the Fermi/LAT results. Without 
a calculation of the $e$ and $\mu$ Fermi/LAT constraints, compatibility of the two experimental results cannot be checked, though the Fermi/LAT constraints are almost certainly too weak to exclude these two points. The $e$ channel is a poor fit to the AMS-02 data and thus of little interest. However, the 4- $\mu$ channel both provides a good fit to the AMS-02 data and escapes Fermi/LAT constraints, thus remaining a viable model.

Reference [57] considered the mixed 4-lepton case, with the best-fit $\langle\sigma v\rangle$ shown by the dotted red curve in Figure 3, using the MED propagation model. The best-fit BRs are annihilation exclusively to taus for DM masses above 2 TeV, and a mix of electrons and taus below that, though still dominated by the tau channel for the masses shown. The Fermi/LAT constraint for these best-fit BRs is shown by the dotted blue curve, which becomes identical to the singlechannel 4- $\tau$ constraint above $2 \mathrm{TeV}$. The AMS-02 best-fit $\langle\sigma v\rangle$ and BRs are incompatible with the Fermi/LAT data except at very high masses $\left(m_{\chi}>40 \mathrm{TeV}\right)$. The analysis shown in Figure 3 includes the case featured by Ref. [57] as their favored scenario: a combination between the four-tau (75\%) and four-electron (25\%) channels for a DM mass between 0.5 and $1 \mathrm{TeV}$. This case is ruled out by Fermi/LAT as an explanation of AMS-02 data (for $m_{\chi}<40$ TeV). The caveats discussed for the $b \bar{b}+\ell \bar{\ell}$ case previously apply here: allowing the BRs to vary from their best-fit values will change the Fermi/LAT constraints and could potentially bring these experimental results into line for this model. The $\tau$ channel would need to be strongly suppressed for that to occur, which implies BRs far different than the best-fit case. An example where this happens is the predominantly $4-\mu$ case which remains a viable model.

In addition to bounds from $\gamma$-rays and antiprotons already discussed, there are other observational constraints on a DM interpretation of the $e^{+}$excess seen in AMS-02. DM annihilation products also include neutrinos. References 70 72 pointed out that a leptophilic explanation of a positron excess should also produce large numbers of neutrinos detectable in the IceCube neutrino observatory. Currently IceCube upper limits are in tension with the DM bestfit to the positron anomaly, assuming MED propagation parameters, when DM annihilates into $W^{+} W^{-} 73$. In addition, measurements of the CMB temperature and polarization provide constraints on the annihilation cross section of DM 74 79. In the near future, papers from the Planck Satellite with extremely strong bounds are expected and could rule out the DM scenario completely.

In summary, we have used the Fermi/LAT dwarf galaxy data in order to constrain dark matter as an explanation of the positron excess seen in HEAT, PAMELA, and AMS-02. In particular, this paper has focused on the annihilation channels that best fit the current AMS-02 data [57. We first considered the single channel case of DM annihilating directly to $b \bar{b}$ and leptons $\ell \bar{\ell}$, as well as to 4-leptons (via a mediator $\phi$ ); we then considered the multi-channel case where annihilation proceeds through a combination of channels. We used $b \bar{b}$ as our proxy for all other quarks, gauge bosons, and Higgs bosons, since the spectra and amplitudes are similar, but considered each lepton flavor separately. For the single channel case, we found that dark matter annihilation into $\left\{b \bar{b}, e^{+} e^{-}, \mu^{+} \mu^{-}, \tau^{+} \tau^{-}, 4-e\right.$, or $\left.4-\tau\right\}$ cannot both provide a good fit to AMS-02 and avoid the $2 \sigma$ upper limit from Fermi/LAT. The AMS-02 best-fit BRs and $\langle\sigma v\rangle$ used for the analysis of this paper assume either the MED propagation model defined in Refs. 68, 69, or the best-fit propagation parameters [57]. Multi-channel annihilations are also highly constrained by Fermi/LAT's measurement of $\gamma$-ray flux. We use the MED propagation model parameters for the mixed channel cases, but doubt that other reasonable choices of CR propagation parameters would change our results. Specifically, we find that the Fermi/LAT $2 \sigma$ upper limits, assuming the best-fit AMS-02 BRs, exclude the annihilation into a combination of $b \bar{b}$ and the three leptons for DM masses $600 \mathrm{GeV} \leq m_{\chi} \leq 100 \mathrm{TeV}$. In addition, the Fermi/LAT upper limit is incompatible with the AMS-02 best fit $\langle\sigma v\rangle$ and BRs for annihilation into a mix of the mediator driven 4-lepton channels except for very high masses $\left(m_{\chi} \geq 40 \mathrm{TeV}\right)$. However, this does not rigorously imply that the results from AMS-02 and Fermi/LAT are in strong conflict for all multi-channel cases, as the Fermi/LAT constraints will vary if the BRs are allowed to deviate from their best fit AMS-02 values. In order to reconcile both experiments, the BRs considered would have to deviate significantly from their best-fit values. We find that the dark matter annhiliation into $4-\mu$ provides a good fit to the AMS-02 data and escapes Fermi/LAT constraints. The reason for the 4 - $\mu$ channel escaping Fermi/LAT upper limits is that it provides positrons in abundance for the AMS-02 signal, while photon production is suppressed. Hence, for the best-fit BR to the AMS-02 data, we find only one viable DM annihilation channel that survives Fermi/LAT constraints and provides a good fit to the current AMS-02 data: the 4- $\mu$ channel.

We briefly mention ways around these conclusions. First, for the mixed channel cases we have only studied the best fits to AMS-02 data (found in Ref. [57]). It is possible that there are other BRs that still provide reasonably good fits (though not the best fits) to the AMS-02 data that are not ruled out by Fermi/LAT bounds from dwarf spheroidals. A joint statistical analysis of the AMS-02 and Fermi/LAT would be required to check for other alternatives. Second, although it is extremely unlikely that the boost factor required by the AMS-02 data is due to a nearby clump of DM that is 100-1000 times as dense as its surroundings, perhaps part of the boost factor is due to a clump of, say, a factor of 10 (again, unlikely). In that case the required annihilation cross-section to explain the AMS-02 data could be lower, and more channels would remain viable. Third, it is possible that part of the AMS-02 signal is due to pulsars, and part due to DM annihilation. Again, more DM annihilation channels might then still remain compatible with Fermi/LAT bounds from dwarf spheroidals. These latter two caveats would also apply to bounds on these scenarios from the CMB including those expected from upcoming Planck data. 


\section{Acknowledgments}

A.L. acknowledges support from the DOE under grant DOE-FG02-95ER40899 and from the Michigan Center for Theoretical Physics. A.L. and D.S. are grateful for financial support from the Swedish Research Council (VR) through the Oskar Klein Centre.

[1] G. Jungman, M. Kamionkowski and K. Griest, Phys. Rept. 267, 195 (1996) hep-ph/9506380.

[2] G. Bertone, D. Hooper and J. Silk, Phys. Rept. 405, 279 (2005) hep-ph/0404175.

[3] M. Srednicki, K. A. Olive and J. Silk, Nucl. Phys. B 279, 804 (1987).

[4] K. Freese, Phys. Lett. B 167, 295 (1986).

[5] L. M. Krauss, M. Srednicki and F. Wilczek, Phys. Rev. D 33, 2079 (1986).

[6] J. R. Ellis, R. A. Flores, K. Freese, S. Ritz, D. Seckel and J. Silk, Phys. Lett. B 214, 403 (1988).

[7] M. S. Turner and F. Wilczek, Phys. Rev. D 42, 1001 (1990).

[8] M. Kamionkowski and M. S. Turner, Phys. Rev. D 43, 1774 (1991).

[9] J. Silk and M. Srednicki, Phys. Rev. Lett. 53, 624 (1984).

[10] L. Bergstrom, P. Ullio and J. H. Buckley, Astropart. Phys. 9, 137 (1998) astro-ph/9712318.

[11] N. W. Evans, F. Ferrer and S. Sarkar, Phys. Rev. D 69, 123501 (2004) astro-ph/0311145.

[12] L. Bergstrom and D. Hooper, Phys. Rev. D 73, 063510 (2006) hep-ph/0512317.

[13] G. Bertone, A. R. Zentner and J. Silk, Phys. Rev. D 72, 103517 (2005) astro-ph/0509565.

[14] P. Sandick, J. Diemand, K. Freese and D. Spolyar, JCAP 1101, 018 (2011) arXiv:1008.3552 [astro-ph.CO]].

[15] P. Sandick, J. Diemand, K. Freese and D. Spolyar, Phys. Rev. D 85, 083519 (2012) arXiv:1108.3820 [astro-ph.CO]].

[16] J. Lavalle and P. Salati, Comptes Rendus Physique 13, 740 (2012) arXiv:1205.1004 [astro-ph.HE]].

[17] L. Accardo et al. [AMS Collaboration], Phys. Rev. Lett. 113, no. 12, 121101 (2014).

[18] O. Adriani et al. [PAMELA Collaboration], Nature 458, 607 (2009) arXiv:0810.4995 [astro-ph]].

[19] O. Adriani et al. [PAMELA Collaboration], Phys. Rev. Lett. 111, no. 8, 081102 (2013) arXiv:1308.0133 [astro-ph.HE]].

[20] F. Collaboration, arXiv:0905.0025 [astro-ph.HE].

[21] S. W. Barwick et al. [HEAT Collaboration], Astrophys. J. 482, L191 (1997) arXiv:astro-ph/9703192].

[22] M. A. DuVernois, S. W. Barwick, J. J. Beatty, A. Bhattacharyya, C. R. Bower, C. J. Chaput, S. Coutu and G. A. de Nolfo et al., Astrophys. J. 559, 296 (2001).

[23] J. J. Beatty, A. Bhattacharyya, C. Bower, S. Coutu, M. A. DuVernois, S. McKee, S. A. Minnick and D. Muller et al., Phys. Rev. Lett. 93, 241102 (2004) astro-ph/0412230.

[24] E. A. Baltz, J. Edsjo, K. Freese and P. Gondolo, Phys. Rev. D 65, 063511 (2002) astro-ph/0109318.

[25] V. Barger, W. Y. Keung, D. Marfatia and G. Shaughnessy, Phys. Lett. B 672, 141 (2009) arXiv:0809.0162 [hep-ph]].

[26] R. Harnik and G. D. Kribs, Phys. Rev. D 79, 095007 (2009) arXiv:0810.5557 [hep-ph]].

[27] M. Cirelli, M. Kadastik, M. Raidal and A. Strumia, Nucl. Phys. B 813, 1 (2009) [Addendum-ibid. B 873, 530 (2013)] arXiv:0809.2409 [hep-ph]].

[28] A. E. Nelson and C. Spitzer, JHEP 1010, 066 (2010) arXiv:0810.5167 [hep-ph]].

[29] I. Cholis, D. P. Finkbeiner, L. Goodenough and N. Weiner, JCAP 0912, 007 (2009) arXiv:0810.5344 [astro-ph]].

[30] K. M. Zurek, Phys. Rev. D 79, 115002 (2009) arXiv:0811.4429 [hep-ph]].

[31] P. J. Fox and E. Poppitz, Phys. Rev. D 79, 083528 (2009) arXiv:0811.0399 [hep-ph]].

[32] C. R. Chen and F. Takahashi, JCAP 0902, 004 (2009) arXiv:0810.4110 [hep-ph]].

[33] I. Cholis, G. Dobler, D. P. Finkbeiner, L. Goodenough and N. Weiner, Phys. Rev. D 80, 123518 (2009) arXiv:0811.3641 [astro-ph]].

[34] D. Hooper and K. M. Zurek, arXiv:0902.0593 [hep-ph].

[35] I. Cholis and D. Hooper, Phys. Rev. D 89, no. 4, 043013 (2014) arXiv:1312.2952 [astro-ph.HE]].

[36] L. Bergstrom, T. Bringmann, I. Cholis, D. Hooper and C. Weniger, Phys. Rev. Lett. 111, 171101 (2013) arXiv:1306.3983 [astro-ph.HE]].

[37] I. Cholis and D. Hooper, Phys. Rev. D 88, 023013 (2013) arXiv:1304.1840 [astro-ph.HE]].

[38] D. Hooper and T. M. P. Tait, Phys. Rev. D 80, 055028 (2009) arXiv:0906.0362 [hep-ph]].

[39] D. Hooper and G. D. Kribs, Phys. Rev. D 70, 115004 (2004) hep-ph/0406026.

[40] D. Hooper, J. E. Taylor and J. Silk, Phys. Rev. D 69, 103509 (2004) hep-ph/0312076.

[41] A. Ibarra, A. S. Lamperstorfer and J. Silk, Phys. Rev. D 89, 063539 (2014) arXiv:1309.2570 [hep-ph]].

[42] I. Cholis, L. Goodenough, D. Hooper, M. Simet and N. Weiner, Phys. Rev. D 80, 123511 (2009) arXiv:0809.1683 [hep-ph]].

[43] P. S. B. Dev, D. K. Ghosh, N. Okada and I. Saha, Phys. Rev. D 89, no. 9, 095001 (2014) arXiv:1307.6204 [hep-ph]].

[44] A. Arvanitaki, S. Dimopoulos, S. Dubovsky, P. W. Graham, R. Harnik and S. Rajendran, Phys. Rev. D 79, 105022 (2009) arXiv:0812.2075 [hep-ph]].

[45] E. Nardi, F. Sannino and A. Strumia, JCAP 0901, 043 (2009) arXiv:0811.4153 [hep-ph]].

[46] A. Arvanitaki, S. Dimopoulos, S. Dubovsky, P. W. Graham, R. Harnik and S. Rajendran, Phys. Rev. D 80, 055011 (2009) arXiv:0904.2789 [hep-ph]].

[47] L. Feng, R. Z. Yang, H. N. He, T. K. Dong, Y. Z. Fan and J. Chang, Phys. Lett. B 728, 250 (2014) arXiv:1303.0530 
[astro-ph.HE]].

[48] P. Brun, T. Delahaye, J. Diemand, S. Profumo and P. Salati, Phys. Rev. D 80, 035023 (2009) arXiv:0904.0812 [astroph.HE]].

[49] O. Adriani, G. C. Barbarino, G. A. Bazilevskaya, R. Bellotti, M. Boezio, E. A. Bogomolov, L. Bonechi and M. Bongi et al., Phys. Rev. Lett. 102, 051101 (2009) arXiv:0810.4994 [astro-ph]].

[50] F. Donato, D. Maurin, P. Brun, T. Delahaye and P. Salati, Phys. Rev. Lett. 102, 071301 (2009) arXiv:0810.5292 [astro-ph]].

[51] O. Adriani et al. [PAMELA Collaboration], Phys. Rev. Lett. 105, 121101 (2010) arXiv:1007.0821 [astro-ph.HE]].

[52] O. Adriani, G. A. Bazilevskaya, G. C. Barbarino, R. Bellotti, M. Boezio, E. A. Bogomolov, V. Bonvicini and M. Bongi et al., JETP Lett. 96, 621 (2013) [Pisma Zh. Eksp. Teor. Fiz. 96, 693 (2012)].

[53] D. Hooper, P. Blasi and P. D. Serpico, JCAP 0901, 025 (2009) arXiv:0810.1527 [astro-ph]].

[54] S. Profumo, Central Eur. J. Phys. 10, 1 (2011) arXiv:0812.4457[ [astro-ph]].

[55] T. Linden and S. Profumo, Astrophys. J. 772, 18 (2013) arXiv:1304.1791 [astro-ph.HE]].

[56] T. Delahaye, K. Kotera and J. Silk, Astrophys. J. 794, no. 2, 168 (2014) arXiv:1404.7546 [astro-ph.HE]].

[57] M. Boudaud, S. Aupetit, S. Caroff, A. Putze, G. Belanger, Y. Genolini, C. Goy and V. Poireau et al., arXiv:1410.3799 [astro-ph.HE].

[58] S. J. Lin, Q. Yuan and X. J. Bi, arXiv:1409.6248 [astro-ph.HE].

[59] M. Ackermann et al. [Fermi-LAT Collaboration], Phys. Rev. D 89, no. 4, 042001 (2014) arXiv:1310.0828 [astro-ph.HE]].

[60] E. Aliu et al. [VERITAS Collaboration], Phys. Rev. D 85, 062001 (2012) arXiv:1202.2144 [astro-ph.HE]].

[61] J. Aleksi, S. Ansoldi, L. A. Antonelli, P. Antoranz, A. Babic, P. Bangale, U. B. de Almeida and J. A. Barrio et al., JCAP 1402, 008 (2014) arXiv:1312.1535[hep-ph]].

[62] A. Abramowski et al. [HESS Collaboration], arXiv:1410.2589 [astro-ph.HE].

[63] J. F. Navarro, C. S. Frenk and S. D. M. White, Astrophys. J. 490, 493 (1997) astro-ph/9611107.

[64] A. Burkert, IAU Symp. 171, 175 (1996) [Astrophys. J. 447, L25 (1995)] astro-ph/9504041.

[65] L. E. Strigari, S. M. Koushiappas, J. S. Bullock, M. Kaplinghat, J. D. Simon, M. Geha and B. Willman, Astrophys. J. 678, 614 (2008) arXiv:0709.1510 [astro-ph]].

[66] T. Sjostrand, S. Mrenna and P. Z. Skands, Comput. Phys. Commun. 178, 852 (2008) arXiv:0710.3820 [hep-ph]].

[67] T. Sjostrand, S. Mrenna and P. Z. Skands, JHEP 0605, 026 (2006) hep-ph/0603175.

[68] F. Donato, N. Fornengo, D. Maurin and P. Salati, Phys. Rev. D 69, 063501 (2004) astro-ph/0306207.

[69] D. Maurin, F. Donato, R. Taillet and P. Salati, Astrophys. J. 555, 585 (2001) astro-ph/0101231.

[70] P. Sandick, D. Spolyar, M. R. Buckley, K. Freese and D. Hooper, Phys. Rev. D 81, 083506 (2010) arXiv:0912.0513 [astro-ph.CO]].

[71] D. Spolyar, M. R. Buckley, K. Freese, D. Hooper and H. Murayama, arXiv:0905.4764 [astro-ph.CO].

[72] A. E. Erkoca, M. H. Reno and I. Sarcevic, Phys. Rev. D 82, 113006 (2010) arXiv:1009.2068 [hep-ph]].

[73] M. G. Aartsen et al. [IceCube Collaboration], Phys. Rev. D 88, no. 12, 122001 (2013) arXiv:1307.3473 [astro-ph.HE]].

[74] S. Galli, F. Iocco, G. Bertone and A. Melchiorri, Phys. Rev. D 80, 023505 (2009) arXiv:0905.0003 [astro-ph.CO]].

[75] G. Giesen, J. Lesgourgues, B. Audren and Y. Ali-Haimoud, JCAP 1212, 008 (2012) arXiv:1209.0247][astro-ph.CO]].

[76] T. R. Slatyer, N. Padmanabhan and D. P. Finkbeiner, Phys. Rev. D 80, 043526 (2009) arXiv:0906.1197 [astro-ph.CO]].

[77] D. P. Finkbeiner, S. Galli, T. Lin and T. R. Slatyer, Phys. Rev. D 85, 043522 (2012) [arXiv:1109.6322 [astro-ph.CO]].

[78] A. Natarajan, Phys. Rev. D 85, 083517 (2012) arXiv:1201.3939 [astro-ph.CO]].

[79] J. M. Cline and P. Scott, JCAP 1303, 044 (2013) [Erratum-ibid. 1305, E01 (2013)] arXiv:1301.5908 [astro-ph.CO]]. 